\title{
Efficacy of trabectedin in advanced soft tissue sarcoma: beyond lipo- and leiomyosarcoma
}

\author{
This article was published in the following Dove Press journal: \\ Drug Design, Development and Therapy \\ 27 October 2015 \\ Number of times this article has been viewed
}

\author{
Rita De Sanctis' \\ Andrea Marrari' \\ Silvia Marchetti' \\ Chiara Mussi² \\ Luca Balzarini ${ }^{3}$ \\ Fabio Romano Lutman ${ }^{3}$ \\ Primo Daolio 4 \\ Stefano Bastoni ${ }^{4}$ \\ Alexia Francesca Bertuzzi ${ }^{1,5}$ \\ Vittorio Quagliuolo² \\ Armando Santoro' \\ 'Department of Medical Oncology and \\ Haematology, ${ }^{2}$ Department of Surgical \\ Oncology, ${ }^{3}$ Department of Radiology, \\ Humanitas Cancer Center, IRCCS, \\ Rozzano, ${ }^{4}$ Department of Surgical \\ Oncology, Orthopaedic Institute \\ "G. Pini", Milan, Italy; ${ }^{5}$ Department of \\ Medical Oncology, Adelaide and Meath \\ Hospital, Incorporating the National \\ Children's Hospital (AMNCH), \\ Dublin, Ireland
}

Correspondence: Rita De Sanctis Humanitas Cancer Center, IRCCS, Via Manzoni, 56, 20089 Rozzano, Milan, Italy Tel +390282247230

Fax +39028224 4590

Email rita.de_sanctis@cancercenter. humanitas.it
Objective: Trabectedin is effective in leiomyosarcoma and liposarcoma, especially the myxoid variant, related to the presence of the FUS-CHOP transcript. We evaluated the efficacy of trabectedin in specific subgroups of patients with soft tissue sarcomas (STS).

Methods: Seventy-two patients with advanced anthracycline-pretreated STS, who received trabectedin at a dose of $1.5 \mathrm{mg} / \mathrm{m}^{2}$ every 3 weeks by continuous 24-hour infusion, were retrospectively analyzed. Best response rate according to Response Evaluation Criteria In Solid Tumors (RECIST) criteria and severe adverse events (AEs) according to National Cancer Institute Common Terminology Criteria for Adverse Events (NCI-CTCAE v4.02) were evaluated. Secondary endpoints included progression-free survival and overall survival (OS).

Results: Median age was 48 (range, 20-75) years, with a median Eastern Cooperative Oncology Group performance status of 0 . The median number of previous chemotherapy regimens was 1 (range, 0-5). Median number of trabectedin cycles was 3 (range, 1-17). About 69/72 patients (95.8\%) were evaluable for response: 9 patients (13\%) achieved partial response and $26(37.7 \%)$ stable disease. According to histotype, clinical benefit (partial response + stable disease) was reported in synovial sarcoma $(n=5)$, retroperitoneal liposarcoma $(n=10)$, myxoid liposarcoma $(n=5)$, leiomyosarcoma $(n=8)$, high-grade undifferentiated pleomorphic sarcoma $(n=5)$, Ewing/ peripheral primitive neuroectodermal tumor $(n=1)$, and malignant peripheral nerve sheath tumor $(n=1)$. Any grade AEs were noncumulative, reversible, and manageable. G3/G4 AEs included anemia $(n=1,1.4 \%)$, neutropenia $(n=7,9.6 \%)$, liver toxicity $(n=6,8.3 \%)$, and fatigue $(n=2,2.8 \%)$. With a median follow-up time of 11 (range, 2-23) months, median progression-free survival and OS of the entire cohort were 2.97 months and 16.5 months, respectively.

Conclusion: Our experience confirms trabectedin as an effective therapeutic option for metastatic lipo- and leiomyosarcoma and suggests promise in synovial sarcomas and high-grade undifferentiated pleomorphic sarcoma.

Keywords: trabectedin, soft tissue sarcoma, liposarcoma, leiomyosarcoma

\section{Introduction}

Trabectedin (Yondelis ${ }^{\circledR}$, Pharma Mar, Madrid, Spain), a novel tetrahydroisoquinoline alkaloid, is a marine antineoplastic agent registered in Europe and other countries for the treatment of adult patients with advanced soft tissue sarcoma (STS), after failure of anthracyclines and ifosfamide, or in patients who are unsuitable to receive these agents. The compound was originally extracted from the Caribbean sea squirt Ecteinascidia turbinata and is now produced synthetically by PharmaMar. Trabectedin is a DNA-binding agent with multiple mechanisms of action based on binding to the minor groove of the DNA double helix, leading to a cascade of events that interfere with several transcription factors, DNA-binding proteins, and DNA-repair pathways, resulting in $\mathrm{G}_{2} / \mathrm{M}$ cell cycle arrest and ultimately apoptosis. ${ }^{1}$ The mechanisms of action of trabectedin, together with clinical evidence and the in vitro demonstration 
of selective cytotoxicity to human monocytes and inhibition of a number of functionally relevant proinflammatory cytokines, suggest that trabectedin acts as multitarget agent on the tumor microenvironment and directly inhibits tumor cell proliferation. ${ }^{1}$

Although trabectedin has shown cytotoxic activity against a range of STS, ovarian cancer, metastatic castration-resistant prostate cancer, and advanced breast cancer, ${ }^{2}$ it appears to be especially active in patients with liposarcoma, specifically myxoid, and leiomyosarcoma. ${ }^{2-8}$ Myxoid liposarcomas and some other STS subtypes, including synovial sarcoma and Ewing's sarcoma, are characterized by the presence of chromosomal translocations, ${ }^{9}$ and there is evidence that trabectedin may have a highly specific mechanism of action in translocationrelated sarcomas by displacing the product of chimeric fusion oncoproteins critical for disease pathogenesis and tumor progression. ${ }^{10} \mathrm{Here}$, we review our institutional series of patients with advanced STS treated with trabectedin to evaluate and compare its efficacy in specific histologic subgroups of STS.

\section{Materials and methods}

This was a retrospective analysis of all patients with advanced anthracycline-pretreated STS treated with trabectedin at Humanitas Cancer Center between March 2008 and May 2014. Patient medical records were reviewed to collect tumor response, treatment outcome, and safety data in specific histological subgroups.

Consecutive patients with histologically confirmed STS, previously treated with at least one anthracycline-based chemotherapy regimen, and with radiological and measurable evidence of progressive disease were included in the study. Patients were aged over 18 years, with an Eastern Cooperative Oncology Group (ECOG) performance status (PS) $\leq 3$, life expectancy $>3$ months, absolute neutrophil count $>1,500 / \mathrm{mm}^{3}$, platelet count $>100,000 / \mathrm{mm}^{3}$, hemoglobin levels $\geq 9 \mathrm{~g} / \mathrm{dL}$, normal liver function defined as normal bilirubin levels, and aspartate aminotransferase and alanine aminotransferase levels less than 2.5 times the upper limit of normal.

Pretreatment evaluation included full medical history, previous treatments, complete physical examination, blood count, and a serum biochemistry panel, testing for hepatitis $\mathrm{B}$ and $\mathrm{C}$ virus, a contrast chest and abdomen computed tomography (CT) scan, or chest CT scan plus abdomen magnetic resonance imaging for patients with renal failure (CT contrast medium not administrable).

The institutional review board of Humanitas Cancer Center (IRCCS, Rozzano, Italy) approved the study. All procedures were conducted in accordance with the Declaration of Helsinki and local ethics regulations. Written informed consent to treatment and to the use of clinical data for scientific purposes had been provided by all patients at the time of chemotherapy administration.

Trabectedin was administered at a dose of $1.5 \mathrm{mg} / \mathrm{m}^{2}$ (with a total top dose of $2.6 \mathrm{mg}$ ) by continuous 24-hour intravenous infusion through a central venous access every 3 weeks. Weekly biochemistry and blood count tests were performed during the intercycle. No dose reduction was initially planned for reversible noncumulative transaminitis. The dose was reduced to $1.2 \mathrm{mg} / \mathrm{m}^{2}$ in case of grade 4 neutropenia lasting more than 5 days, neutropenic fever, grade 4 thrombocytopenia, bilirubin increase, or 3-week-lasting transaminitis and was reduced to $1.0 \mathrm{mg} / \mathrm{m}^{2}$ in case of further bone marrow and/or liver toxicity. Any patient requiring a dose reduction continued to receive a reduced dose for the remainder of the treatment.

Premedication against hepatic toxicity and as antiemetic prophylaxis consisted of dexamethasone $4 \mathrm{mg}$ orally twice daily on days $-1,+1$, and +2 . In addition, an intravenous 5-hydroxytryptamine- 3 antagonist and an intravenous bolus of $20 \mathrm{mg}$ of dexamethasone were also administered approximately 20 minutes before the start of trabectedin infusion.

Granulocyte colony-stimulating factor and erythropoiesisstimulating agents were permitted only in accordance with American Society of Clinical Oncology guidelines. ${ }^{11,12}$

Treatment continued until disease progression or discontinuation for other reasons, such as unacceptable toxicity or consent withdrawal.

The primary objective of the study was to evaluate best response rate according to Response Evaluation Criteria in Solid Tumors (RECIST) criteria version $1.1^{13}$ and incidence of severe adverse events (AEs) according to the National Cancer Institute Common Terminology Criteria for Adverse Events (NCI-CTCAE) version 4.02.14

Secondary objectives included Kaplan-Meier estimates of progression-free survival (PFS) and overall survival (OS) and response evaluation defined by the growth modulation index (GMI) ${ }^{15}$ induced by trabectedin. GMI was calculated as the ratio between PFS after trabectedin treatment $\left(\mathrm{PFS}_{\mathrm{T}}\right)$ and PFS after first/previous treatment line $\left(\mathrm{PFS}_{1}\right)$ in each single patient.

Disease evaluations were carried out at baseline (predose, but not more than 3 weeks before initiating treatment with trabectedin), after the first two cycles, and then every three cycles, according to regulatory indications. Response analyses included all patients who received at least one dose of trabectedin and for whom a radiological response evaluation was available. After completion of treatment, patients 
were similarly evaluated every 3 months until disease progression. Patients were also observed for survival until death or last contact if still alive.

\section{Statistical analysis}

The study was planned as a retrospective evaluation of treatment with trabectedin in a consecutive series of pretreated patients. Actuarial survival curves were generated using the Kaplan-Meier method. Median follow-up time was estimated with the use of the inverse Kaplan-Meier method. PFS was defined as the time from the first day of study treatment until disease progression, as shown by radiological or clinical examination, or death from any cause. Patients without any evidence of progressive disease were censored at the date of their last follow-up. OS was calculated as the time from the first day of study treatment until death from any cause; patients who were alive on the date of their last follow-up were censored on that date. We estimated the correlation coefficient between $\mathrm{PFS}_{1}$ and $\mathrm{PFS}_{\mathrm{T}}$. The exploration of the link between GMI and OS was carried out using log-rank test. PFS and OS after treatment with trabectedin were also analyzed according to ECOG PS, age, histology, sex and line of therapy, and response. Differences between strata were evaluated using the log-rank test. Statistical significance was set at 0.05 , and all analyses were performed with the R software package.

\section{Results}

\section{Patient population}

A total of 72 patients with advanced, anthracycline-pretreated STS were treated.

Demographic and clinical characteristics are summarized in Table 1. The male/female ratio was 1:1. The median age was 48 years (range, 20-75 years). Most had an ECOG PS of 0 or $1(88.9 \%)$. Median number of chemotherapy regimens in the metastatic setting before trabectedin was 1 (range, $0-5$ ). Three patients had been enrolled in a randomized Phase III study comparing trabectedin to doxorubicin-based chemotherapy as first line in patients with metastatic sarcoma. ${ }^{16}$

A median of three cycles of trabectedin per patient were administered (range, 1-17).

\section{Efficacy}

Sixty-nine patients were evaluable for response. Three patients stopped treatment before the first evaluation: one died of rapid progressive disease and two stopped chemotherapy due to persistent liver toxicity. All of these patients were considered to have progressed according to an intentionto-treat-analysis.
Table I Baseline demographic and clinical characteristics

\begin{tabular}{|c|c|}
\hline Characteristic & $\begin{array}{l}\text { Patients } \\
(\mathrm{N}=72)\end{array}$ \\
\hline Age, median (range), years & $48(20-75)$ \\
\hline \multicolumn{2}{|l|}{ Sex, n (\%) } \\
\hline Female & $37(5 \mid .4)$ \\
\hline Male & $35(48.6)$ \\
\hline \multicolumn{2}{|c|}{ Eastern Cooperative Oncology Group status, n (\%) } \\
\hline 0 & $37(5 \mid .4)$ \\
\hline I & $27(37.5)$ \\
\hline 2 & $8(11.1)$ \\
\hline \multicolumn{2}{|l|}{ Number of prior chemotherapy regimens, $\mathrm{n}(\%)$} \\
\hline$\leq 2$ lines & $60(83.3)$ \\
\hline 3 lines & $7(9.7)$ \\
\hline$\geq 4$ lines & $5(7)$ \\
\hline \multicolumn{2}{|l|}{ Tumor histology, n (\%) } \\
\hline Leiomyosarcoma & $20(27.8)$ \\
\hline Liposarcoma & $21(29.2)$ \\
\hline Synovial sarcoma & $10(13.9)$ \\
\hline HGUPS & $9(12.5)$ \\
\hline Ewing sarcoma & $5(6.9)$ \\
\hline MPNST & $3(4.1)$ \\
\hline Fibrosarcoma & $2(2.8)$ \\
\hline Desmoplastic small round cell tumor & $\mathrm{I}(\mathrm{I} .4)$ \\
\hline Endometrial stromal sarcoma & $\mathrm{I}(\mathrm{I} .4)$ \\
\hline
\end{tabular}

Abbreviations: HGUPS, high-grade undifferentiated pleomorphic sarcoma; MPNST, malignant peripheral nerve sheath tumor.

A partial response (PR) was achieved in nine patients, for an objective response rate of $13 \%$. Twenty-six patients $(38 \%)$ were considered to have stable disease (SD), and 34 $(49 \%)$ had progressive disease. No complete response was observed. Overall, the disease control rate (DCR, defined as $\mathrm{PR}+\mathrm{SD}$ ) was $51 \%$. According to histotype-based evaluation (Figure 1), the best responses were recorded among

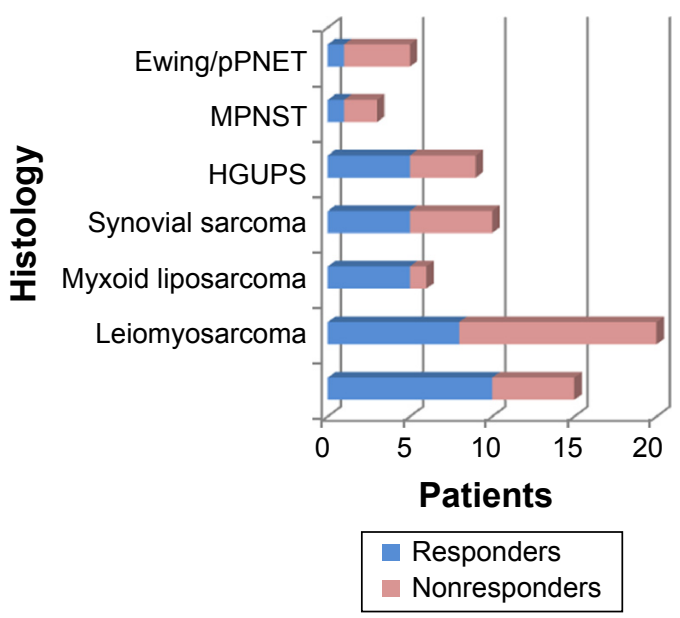

Figure I Clinical benefit (partial response + stable disease; patients classified as responders) and progression (nonresponders) by histotype in patients treated with trabectedin.

Abbreviations: HGUPS, high-grade undifferentiated pleomorphic sarcoma; MPNST, malignant peripheral nerve and sheath tumor; pPNET, peripheral primitive neuroectodermal tumor. 
Table 2 Treatment exposure and efficacy endpoints

\begin{tabular}{|c|c|}
\hline \multicolumn{2}{|l|}{ Treatment exposure $(\mathrm{N}=72)$} \\
\hline Number of trabectedin cycles, median (range) & $3(1-17)$ \\
\hline Follow-up time (months), median (range) & II $(2-43)$ \\
\hline \multicolumn{2}{|l|}{ Best response ${ }^{a}$ in evaluable patients $(\mathrm{N}=69), \mathrm{n}(\%)$} \\
\hline PR & $9(13.1)$ \\
\hline SD & $26(37.6)$ \\
\hline Disease control rate $(P R+S D), \mathrm{n}(\%)$ & $35(50.7)$ \\
\hline Retroperitoneal liposarcoma & $10(14.5)$ \\
\hline Myxoid liposarcoma & $5(7.2)$ \\
\hline Leiomyosarcoma & $8(I 1.6)$ \\
\hline Synovial sarcoma & $5(7.2)$ \\
\hline HGUPS & $5(7.2)$ \\
\hline Ewing sarcoma & I (I.5) \\
\hline MPNST & I (I.5) \\
\hline Response duration (months), mean $(95 \% \mathrm{Cl})$ & $7.4(4.9-9.9)$ \\
\hline
\end{tabular}

retroperitoneal liposarcomas (10 out of all 15 enrolled liposarcomas), myxoid liposarcomas (5/6), leiomyosarcomas $(8 / 20)$, synovial sarcomas $(5 / 10)$, undifferentiated pleomorphic sarcomas (5/9), malignant peripheral nerve sheath tumor $(1 / 3)$, and peripheral primitive neuroectodermal tumor $(1 / 5)$. No response was observed in a case of high-grade endometrial stromal sarcoma. A summary of treatment exposure and efficacy endpoints is presented in Table 2.

With a median follow-up of 11 months (range, 2-43 months), 48 patients had died by the time of the study, whereas 24 were still alive. Median PFS of the entire cohort was 2.97 months (range, 2-42.3 months). Median OS was 16.5 months (range, 0.8-43 months). Kaplan-Meier curves for PFS and OS in the total population are presented in Figures 2 and 3, respectively.

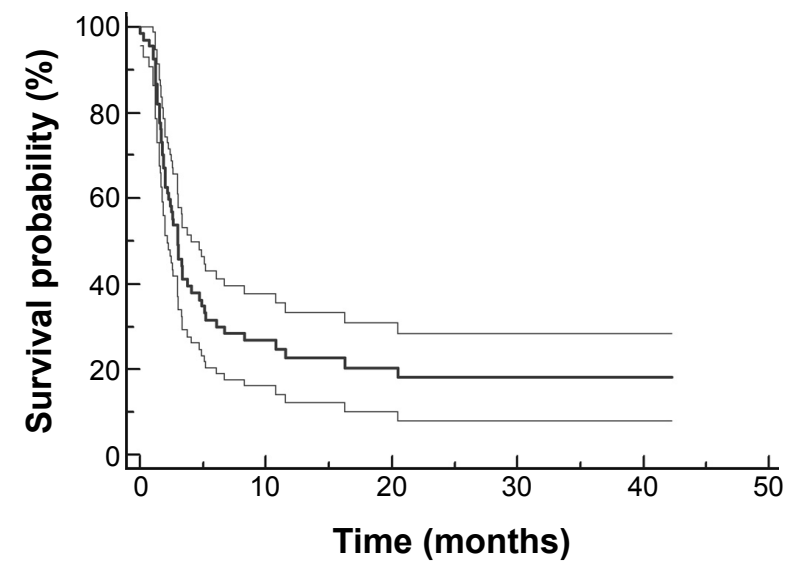

Figure 2 Kaplan-Meier curve for PFS in the entire cohort $(n=72)$.

Notes: The upper and lower (nonbolded) lines are the relative $95 \% \mathrm{Cl}$. The median PFS was 2.97 months ( $95 \% \mathrm{Cl}, 2.0-42.3$ months).

Abbreviations: $\mathrm{Cl}$, confidence intervals; PFS, progression-free survival.

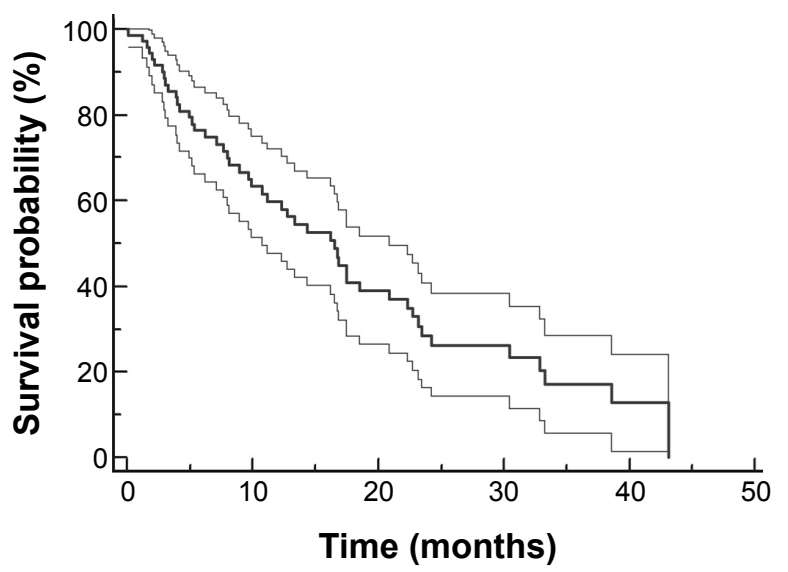

Figure 3 Kaplan-Meier curve for OS in patients treated with trabectedin $(n=72)$. Notes: The upper and lower (nonbolded) lines are the relative $95 \% \mathrm{Cl}$. The median OS was 16.5 months ( $95 \% \mathrm{Cl}, 0.8-43$ months).

Abbreviations: $\mathrm{Cl}$, confidence intervals; OS, overall survival.

The median PFS after PFS $_{1}$ was 4.53 months (range, 0-48.9 months). The median $\mathrm{PFS}_{\mathrm{T}}$ was 2.76 months (range, 2-42 months). The median growth modulation index ( $\mathrm{PFS}_{\mathrm{T}} /$ $\mathrm{PFS}_{1}$ ) was 0.47 (range, 0.02-31.54). Twenty-one patients (29.2\%) experienced a GMI $>1.33$, which was suggested to be a clear sign of drug activity by Von Hoff. ${ }^{15}$ There was a strong correlation between GMI and OS: correlation coefficient $r$ was 0.34 ([95\% confidence interval, 0.12-0.53], $P=0.0036$ ). Considering two subgroup of patients with $\mathrm{GMI} \leq 1.33$ and $\mathrm{GMI}>1.33$, the median OS was 12.27 and 22.33 months, respectively, and GMI $>1.33$ was associated with a significant improvement of OS ( $P=0.03$; Figure 4).

Patients with disease control $(\mathrm{PR}+\mathrm{SD})$ had a longer OS (30.5 vs 6.2 months; $P<0.0001$ ). Patients with an ECOG PS of 0 had a similar DCR to that of patients with higher ECOG PS (51.3\% vs 50\%). No difference was observed in

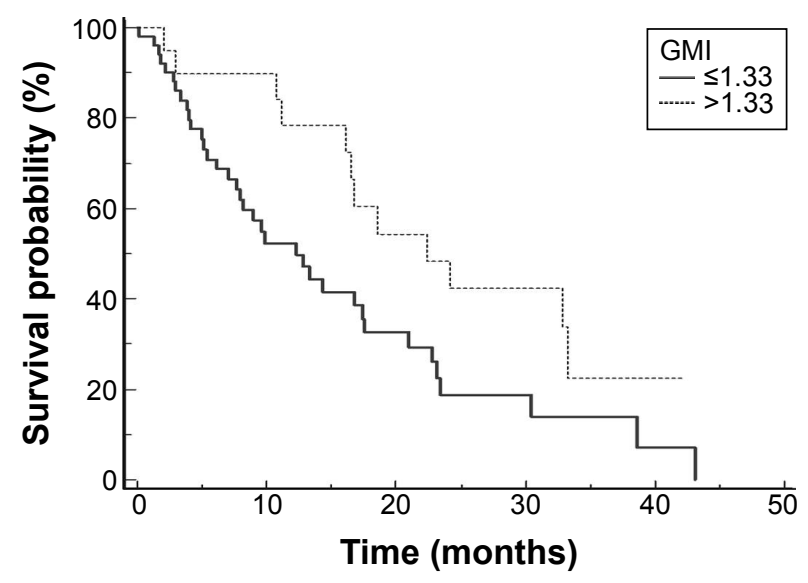

Figure 4 Kaplan-Meier curve for OS in patients treated with trabectedin $(n=72)$, according to their $\mathrm{GMI}(\leq 1.33$ vs $>1.33)$.

Abbreviations: GMI, growth modulation index; OS, overall survival. 
Table 3 Severe (grade 3/4) adverse events according to $\mathrm{NCl}-\mathrm{CTC}$ $(\mathrm{N}=72)$

\begin{tabular}{ll}
\hline Adverse event & Patients, n (\%) \\
\hline Febrile neutropenia & $3(4 . \mathrm{I})$ \\
Afebrile neutropenia & $4(5.5)$ \\
Anemia & $\mathrm{I}(1.4)$ \\
Liver toxicity & $6(8.3)$ \\
Fatigue & $2(2.8)$ \\
Hemothorax & $\mathrm{I}(\mathrm{I} .4)$ \\
\hline
\end{tabular}

Abbreviation: $\mathrm{NCl}-\mathrm{CTC}$, National Cancer Institute Common Terminology Criteria.

terms of PFS or OS stratifying for age, histology, sex, and line of therapy.

\section{Safety}

All 72 patients were included in the safety analysis, and a total of 314 cycles were evaluable for toxicity. Treatment with trabectedin was generally well tolerated, and the toxicity profile of trabectedin in this study was consistent with AEs previously reported. The most common trabectedin-related AEs were liver toxicity, fatigue, and constipation with a global incidence of $83.3 \%, 27.8 \%$, and $18 \%$, respectively. In general, AEs were noncumulative, reversible, and manageable.

Serious AEs are presented in Table 3. Grade 3/4 elevation of aspartate aminotransferase and alanine aminotransferase serum levels was found in six patients $(8.3 \%)$, although it appeared transient and noncumulative. There were no toxic deaths, but two patients discontinued trabectedin due to persistent toxicity over 6 weeks. Dose reduction occurred in 17 patients for grade 4/4 toxicities. Among them, we observed a hemothorax as a complicated response to trabectedin in a patient with undifferentiated pleomorphic sarcoma with lung metastases. A second patient experienced fatigue, which was highly interfering with his daily activities, only partially responding to drug dose reduction and supportive care, so the patient himself withdrew the informed consent to the treatment, notwithstanding a SD observed at the evaluation CT scans.

\section{Discussion}

Our experience in anthracycline-pretreated patients with advanced STS confirms that trabectedin is an effective therapeutic option for patients with metastatic liposarcoma and leiomyosarcoma and that it could also be a promising option for patients with synovial sarcoma and high-grade undifferentiated pleomorphic sarcoma (HGUPS). Of note, HGUPS are a very aggressive neoplasm and relatively poorly chemosensitive.

We showed that trabectedin provided in the entire population a DCR of $51 \%$, with a median PFS of 2.97 months (range, 2-42.3 months) and a median OS of 16.5 months (range, 0.8-43 months). Despite a less rigorous patient selection in a retrospective study and the heterogeneity of histologies, outcomes were consistent with results from previous studies..$^{3,7,16-19}$ Of note, median PFS and OS were higher in responder patients, which could be considered as obvious, but, at the same time, it highlights the urgent need of predictive factors of response to trabectedin.

It has been demonstrated that trabectedin has an encouraging activity in STS subtypes, carrying specific chromosomal translocations, resulting in increased sensitivity to chemotherapeutic agents such as trabectedin.4,6,9,16,17,19,20 Trabectedin has been shown to be effective in prospective and retrospective series of patients with myxoid round cell liposarcoma, ${ }^{4,5}$ which has the translocation $\mathrm{t}(12: 16)(\mathrm{q} 13$; p11) as a genetic hallmark. This translocation produces the chimeric protein FUS-CHOP that binds to specific DNA promoters, leading to deregulated expression of downstream proteins. In vitro, trabectedin interferes with the binding of this fusion protein to DNA promoters. In our cohort, among translocation-related sarcomas $(17 / 72,23.6 \%)$, we observed a DCR of $58.9 \%$, considering synovial sarcomas (DCR, 5/10 patients), myxoid round cell liposarcoma (5/6), and high grade endometrial stromal sarcoma (no response). This data suggest that chimeric oncoproteins deriving from typical sarcoma translocations represent a potential therapeutic target more than a predictive factor. Unfortunately, as for almost all molecular targets in medical oncology, the response to a target therapy depends on the interaction of alternative pathways and their related factors. Indeed, the randomized Phase III trial of trabectedin vs standard doxorubicin-based chemotherapy as first-line therapy in 88 patients with pathologically confirmed translocation-related sarcomas did not show a significant superiority of any treatment. ${ }^{16}$ There was a nonsignificant difference in PFS between groups at the time of analysis; 29 PFS events were assessed, with 16 in the trabectedin group and 13 in doxorubicin-treated patients. There was no statistically significant difference in OS between groups (stratified log-rank test, $P=0.9573$; hazard ratio $=0.86, P=0.6992$ ), although the Kaplan-Meier survival curves after month 20 favored trabectedin, which could be administered for extended periods due to lack of cumulative toxicity. However, the study was underpowered for statistical analyses. Nevertheless, the study confirmed the activity of first-line trabectedin in this patient group of advanced translocation-related sarcoma.

Toward identifying particular patient subgroups that have greater or lesser sensitivity to trabectedin treatment, 
Schöffski et $\mathrm{al}^{21}$ and Italiano et $\mathrm{al}^{22}$ retrospectively investigated the predictive impact of various genetic profiles from the nucleotide excision repair and homologous recombination repair DNA repair pathways in patients with advanced STSs treated with trabectedin. They found a correlation between better response and low BRCA1 (breast cancer susceptibility 1 gene) mRNA expression and high ERCC1 (excision repair cross-complementation group 1 gene) or XPG (xeroderma pigmentosum group G gene) expression in tumor samples, suggesting that these genetic signatures may represent potential biomarkers of response to trabectedin treatment. However, these data have yet to be validated prospectively, and further investigation into the main mechanisms of action of trabectedin at the cellular level and in the tumor microenvironment are required to identify biomarkers predictive of clinical benefit, which would allow the optimization of treatment outcomes through the selection of patients who may largely benefit from trabectedin-based therapies and the avoidance of unnecessary treatment in patients unlikely to respond.

Even on unselected patients within a retrospective analysis, the results of our study provide the opportunity to describe and define the use of trabectedin in patients with worse ECOG PS, both in terms of activity and toxicity. Trabectedin was generally well tolerated: the drug-related treatment discontinuation rate was $2.8 \%$. As expected, the most common toxicities comprised liver toxicity, fatigue, and neutropenia. ${ }^{23}$ This low toxicity profile enabled the administration of more than six cycles of trabectedin in $16.7 \%$ of patients, in accordance with the indication of treating patients until disease progression.

Moreover, our study confirms a correlation between GMI and OS in pretreated STS patients. ${ }^{24,25}$ The use of GMI has been shown in retrospective studies to correlate strongly with classical endpoints used in the assessment of cancer treatments in clinical trials. In our study, a GMI $>1.33$, considered to be an indicator of treatment activity, was associated with a significant improvement of OS (9.06 months, $P=0.03$ ). Therefore, the GMI parameter should be considered as an intrapatient comparator of PFS, with each patient/tumor couple being its own control.

\section{Conclusion}

Trabectedin provided a clinical benefit and an encouraging level of disease control in STS subgroups, with a manageable AEs profile. We consider that our data provide support for future clinical investigation of trabectedin in patients with STS not limited to liposarcomas and leiomyosarcomas, but in other subtypes, including synovial sarcoma and HGUPS.
As the mechanism of action of trabectedin may be related to the presence of specific chromosomal translocations, integrated studies of molecular biology and pharmacology are required to further define the relevant the therapeutic targets and optimal histology-driven treatment strategies for this novel antitumor agent.

\section{Acknowledgment}

The authors thank Ray Hill, an independent medical writer, who provided native English editing and journal styling funded by PharmaMar, Spain.

\section{Disclosure}

The authors report no conflicts of interest in this work.

\section{References}

1. Germano G, Frapolli R, Belgiovine C, et al. Role of macrophage targeting in the antitumor activity of trabectedin. Cancer Cell. 2013; 23(2):249-262.

2. Carter NJ, Keam SJ. Trabectedin: a review of its use in the management of soft tissue sarcoma and ovarian cancer. Drugs. 2007;67(15): 2257-2276.

3. Demetri GD, Chawla SP, von Mehren M, et al. Efficacy and safety of trabectedin in patients with advanced or metastatic liposarcoma or leiomyosarcoma after failure of prior anthracyclines and ifosfamide: results of a randomized phase II study of two different schedules. J Clin Oncol. 2009;27(25):4188-4196.

4. Grosso F, Jones RL, Demetri GD, et al. Efficacy of trabectedin (ecteinascidin-743) in advanced pretreated myxoid liposarcomas: a retrospective study. Lancet Oncol. 2007;8(7):595-602.

5. Grosso F, Sanfilippo R, Virdis E, et al. Trabectedin in myxoid liposarcomas (MLS): a long-term analysis of a single-institution series. Ann Oncol. 2009;20(8):1439-1444.

6. Movva S, Verschraegen C. Systemic management strategies for metastatic soft tissue sarcoma. Drugs. 2011;71(16):2115-2129.

7. Le Cesne A, Ray-Coquard I, Duffaud F, et al. Trabectedin in patients with advanced soft tissue sarcoma: a retrospective national analysis of the French Sarcoma Group. Eur J Cancer. 2015;51(6):742-750.

8. Demetri GD, von Mehren M, Jones RL, et al. A randomized phase III study of trabectedin (T) or dacarbazine (D) for the treatment of patients (pts) with advanced liposarcoma (LPS) or leiomyosarcoma (LMS) [abstract]. J Clin Oncol. 2015;33(Suppl):A10503.

9. Mertens F, Antonescu CR, Hohenberger P, et al. Translocation-related sarcomas. Semin Oncol. 2009;36(4):312-323.

10. Di Giandomenico S, Frapolli R, Bello E, et al. Mode of action of trabectedin in myxoid liposarcomas. Oncogene. 2014;33(44):5201-5210.

11. Rizzo JD, Brouwers M, Hurley P, et al. American Society of Clinical Oncology/American Society of Hematology clinical practice guideline update on the use of epoetin and darbepoetin in adult patients with cancer. J Clin Oncol. 2010;28(33):4996-5010.

12. Smith TJ, Khatcheressian J, Lyman GH, et al. 2006 update of recommendations for the use of white blood cell growth factors: an evidence-based clinical practice guideline. J Clin Oncol. 2006;24(19):3187-3205.

13. Eisenhauer EA, Therasse P, Bogaerts J, et al. New response evaluation criteria in solid tumours: revised RECIST guideline (version 1.1). Eur J Cancer. 2009;45(2):228-247.

14. National Institutes of Health/National Cancer Institute. Common Terminology Criteria for Adverse Events (CTCAE) Version 4.03; 2009. Available from: http://www.eortc.be/services/doc/ctc/. Accessed June 27, 2014.

15. Von Hoff DD. There are no bad anticancer agents, only bad clinical trial designs - twenty-first Richard and Hinda Rosenthal Foundation Award Lecture. Clin Cancer Res. 1998;4(5):1079-1086. 
16. Blay JY, Leahy MG, Nguyen BB, et al. Randomised phase III trial of trabectedin versus doxorubicin-based chemotherapy as first-line therapy in translocation-related sarcomas. Eur J Cancer. 2014;50(6): 1137-1147.

17. Le Cesne A, Cresta S, Maki RG, et al. A retrospective analysis of antitumour activity with trabectedin in translocation-related sarcomas. Eur J Cancer. 2012;48(16):3036-3044.

18. Le Cesne A, Judson I, Maki R, et al. Trabectedin is a feasible treatment for soft tissue sarcoma patients regardless of patient age: a retrospective pooled analysis of five phase II trials. Br J Cancer. 2013;109(7): $1717-1724$.

19. Sanfilippo R, Dileo P, Blay JY, et al. Trabectedin in advanced synovial sarcomas: a multicenter retrospective study from four European institutions and the Italian Rare Cancer Network. Anticancer Drugs. 2015;26(6):678-681.

20. Schöffski P, Dumez H, Wolter P, et al. Clinical impact of trabectedin (ecteinascidin-743) in advanced/metastatic soft tissue sarcoma. Exp Opin Pharmacother. 2008;9(9):1609-1618.
21. Schöffski P, Taron M, Jimeno J, et al. Predictive impact of DNA repair functionality on clinical outcome of advanced sarcoma patients treated with trabectedin: a retrospective multicentric study. Eur J Cancer. 2011; 47(7):1006-1012.

22. Italiano A, Laurand A, Laroche A, et al. ERCC5/XPG, ERCC1, and BRCA1 gene status and clinical benefit of trabectedin in patients with soft tissue sarcoma. Cancer. 2011;117(15):3445-3456.

23. Martin-Liberal J, Judson I. Safety evaluation of trabectedin in treatment of soft-tissue sarcomas. Exp Opin Drug Saf. 2013;12(6):905-911.

24. Cousin S, Blay JY, Bertucci F, et al. Correlation between overall survival and growth modulation index in pre-treated sarcoma patients: a study from the French Sarcoma Group. Ann Oncol. 2013;24(10): 2681-2685.

25. Penel N, Demetri GD, Blay JY, et al. Growth modulation index as metric of clinical benefit assessment among advanced soft tissue sarcoma patients receiving trabectedin as a salvage therapy. Ann Oncol. 2013;24(2):537-542.

\section{Publish your work in this journal}

Drug Design, Development and Therapy is an international, peerreviewed open-access journal that spans the spectrum of drug design and development through to clinical applications. Clinical outcomes, patient safety, and programs for the development and effective, safe, and sustained use of medicines are a feature of the journal, which has also been accepted for indexing on PubMed Central. The manuscript management system is completely online and includes a very quick and fair peer-review system, which is all easy to use. Visit http://www.dovepress.com/testimonials.php to read real quotes from published authors.

Submit your manuscript here: http://www.dovepress.com/drug-design-development-and-therapy-journal 